\title{
THE FULL NONRIGID GROUP THEORY FOR TRIMETHYLAMINE
}

\author{
MASOOD HAMADANIAN and ALI REZA ASHRAFI
}

Received 23 May 2002

\begin{abstract}
The nonrigid molecule group theory (NRG) in which the dynamical symmetry operations are defined as physical operations is a new field in chemistry. Smeyers in a series of papers applied this notion to determine the character table of restricted NRG of some molecules. In this note, a simple method is described by means of which it is possible to calculate character tables for the symmetry group of molecules consisting of a number of methyl groups attached to a rigid framework. We study the full NRG of trimethylamine $\mathrm{N}\left(\mathrm{CH}_{3}\right)_{3}$ and prove that it is a group of order 1296 with 28 conjugacy classes. The method can be generalized to apply to other nonrigid molecules. The full nonrigid (f-NRG) molecule group theory is seen to be used advantageously to study the internal dynamics of such molecules.
\end{abstract}

2000 Mathematics Subject Classification: 92E10.

1. Introduction. Group theory for nonrigid molecules (NRG), developed to accommodate two distinct points of view, has grown in popularity recently. Smeyers in [8] and others in [10, 12, 13] list among the areas of application large amplitude vibrations in spectroscopy of small organic molecules.

(I) The molecular symmetry group theory (MSG) of permutation inversion group (PI) is constructed by permutations and permutation-inversion of identical particles. The MSG group is then formed by all feasible permutations and permutations-inversions (see $[1,5]$ ).

In [5], Longuet-Higgins investigated the symmetry groups of nonrigid molecules, where changes from one conformation to another can occur easily. In many cases, these symmetry groups are not isomorphic with any of the familiar symmetry groups of rigid molecules and their character tables are not known. It is therefore of some interest and importance to develop simple methods of calculating these character tables, which are needed for classification of wavefunctions, determination of selection rules, and so on.

Lomont [4] has given two methods for calculating character tables. These are satisfactory for small group, but both of them require a knowledge of the class constant and hence of the group multiplication table and they become very unwieldy as soon as the order of the group becomes even moderately large. For nonrigid molecules, whose symmetry groups may have several thousand elements, they are usually quite impracticable. 
In [11], Stone described a method which is appropriate for molecules with a number of $\mathrm{XH}_{3}$ groups attached to a rigid framework. It is not appropriate in cases where the framework is linear, as in ethane and dimethylacetylene.

(II) The full and restricted nonrigid group theory (f- or r-NRG) built up with physical operations, expressed in terms of internal coordinates that transform one conformation into another one is isoenergetic. The r-NRG is then formed by the complete set of physical operations which commute with given restricted or Hamiltonian operators (for details see [6, 7]).

In [9], Smeyers and Villa investigated the r-NRG of planer trimethylamine and proved that this is a group of order 324. Furthermore, they showed that this molecule has a pyramidal inversion and so the order of r-NRG of trimethylamine is 648 .

In this note, we investigate the f-NRG of pyramidal trimethylamine and prove that this is a group $G$ of order 1296. Also, we show that this group does not have a copy of r-NRG of pyramidal trimethylamine. Throughout this note, all considered groups are assumed to be finite. Our notation is standard and is taken mainly from [2, 3].

2. Determination of conjugacy classes. First of all, we consider the point group of the molecule in the case of rigid state. The point group of pyramidal trimethylamine is $C_{3 v}$. The process of enumerating the symmetry operations of this molecule and arranging them in classes can be explained as adopting a numbering convention for the central atom of molecule, central atom of every $\mathrm{CH}_{3}$ group, and protons nuclei.

Suppose that $H$ is the full nonrigid group of trimethylamine without inversion. It is easy to see that $G \cong Z_{2} \times H$. If we compute the conjugacy classes and character table of $H$, then using a well-known method for calculating conjugacy classes and character tables of direct product of groups, we can complete the character table of $G$. So, it is enough to investigate the conjugacy classes and character table of $H$.

The process of enumerating the symmetry operations of this molecule and arranging them in classes can be explained as adopting a numbering convention for the proton and carbon nuclei. We define the operations $\alpha=(5,6,7)$, $\beta=(8,9,10)$, and $\gamma=(11,12,13)$, which are rotations, in a positive sense for the three methyl groups. We assume that these operations are all feasible, that is, that the barrier to rotation of the methyl group is low.

Now we consider the first operation which leaves the framework of the molecule unchanged. Each methyl group can be left alone or rotated through $120^{\circ}$ in either direction, so that there are $3^{3}=27$ of such operations. These fall into four classes; the class numbers 1-4 of Table 2.1 show a representative of each, together with the number of elements in the class. These results are obtained as follows. First, the operations are grouped according to their cycle structure; operations which rotate different numbers of methyl groups must belong to 
TABLE 2.1. The representatives of conjugacy classes.

\begin{tabular}{rlr}
\hline No. & Representatives & Size \\
\hline 1 & () & 1 \\
2 & $(5,6,7)$ & 6 \\
3 & $(5,6,7)(8,9,10)$ & 12 \\
4 & $(5,6,7)(8,9,10)(11,12,13)$ & 8 \\
5 & $(5,7)(11,13)$ & 27 \\
6 & $(5,7)(8,9,10)(11,13)$ & 54 \\
7 & $(3,4)(8,13)(9,11,10,12)$ & 54 \\
8 & $(3,4)(6,7)(8,11)(9,13)(10,12)$ & 54 \\
9 & $(3,4)(5,7,6)(8,13)(9,11,10,12)$ & 54 \\
10 & $(3,4)(5,6,7)(8,12)(9,13,10,11)$ & 72 \\
11 & $(2,3,4)(5,8,11)(6,9,12)(7,10,13)$ & 72 \\
12 & $(2,3,4)(5,9,13,7,8,12,6,10,11)$ & 72 \\
13 & $(2,3,4)(5,10,12,6,8,13,7,9,11)$ & 108 \\
14 & $(3,4)(6,7)(8,12,10,13,9,11)$ & \\
\hline
\end{tabular}

different conjugacy classes. If now we consider the six operations which rotate one methyl group, we can see that they must all belong to the same class since operations involving rotation of the molecular framework will transform $\alpha$ into $\alpha^{-1}, \beta^{-1}$, or $\gamma^{-1}$, changing the sense of the rotation. All of the operations which rotate two methyl groups and all of the operations which rotate three methyl groups constitute two conjugacy classes of the group.

Consider next the operation which permutes the nuclei of the framework; these fall into sets corresponding to the classes of $C_{3 v}$. One of the $C_{3}$ operations of $C_{3 v}$ applied to the framework is the permutation $(2,3,4)$, but this is not feasible for the molecule as the whole, and one has permutated the protons as well.

We consider the following three permutations:

$$
\begin{aligned}
& R_{1}=(2,3,4)(5,8,11)(6,9,12)(7,10,13), \\
& R_{2}=(2,3,4)(5,9,13,7,8,12,6,10,11), \\
& R_{3}=(2,3,4)(5,10,12,6,8,13,7,9,11) .
\end{aligned}
$$

The permutation $R_{1}$ denotes the case that the protons of each methyl group do not rotate. But $R_{2}$ and $R_{3}$ apply for the case that these protons rotate. Using a tedious calculation, we can see that these permutations are not conjugate and have the same length. Hence, we obtain three conjugacy classes of lengths 72. 
Similar methods are applied to the other classes of $C_{3 v}$ to derive other conjugacy classes for trimethylamine. We now define

$$
\begin{aligned}
& \sigma_{v_{1}}=(3,4)(6,7)(8,11)(9,13)(10,12), \\
& \sigma_{v_{2}}=(3,4)(6,7)(8,12,10,13,9,11) .
\end{aligned}
$$

Then we obtain two conjugacy classes of lengths 54 and 108 with the representatives $\sigma_{v_{1}}$ and $\sigma_{v_{2}}$, respectively. Using similar arguments, we can calculate all of the 14 conjugacy classes of the group $H$. Since $G \cong Z_{2} \times H$, we can see that $G$ has exactly 28 conjugacy classes. In Table 2.1 , we give representatives for the conjugacy classes of $H$. The conjugacy classes of the group $G$ can be computed from this table.

3. Determination of character table. From the conjugacy classes of the group $H$ in Section 2, we can see that $H$ is a group of order 648. First of all, we denote the conjugacy classes of $H$ and their centralizer orders by the following two vectors:

$$
\begin{aligned}
& A=(1 a, 3 a, 3 b, 3 c, 2 a, 6 a, 3 d, 9 a, 9 b, 4 a, 12 a, 12 b, 6 b, 2 b), \\
& B=(648,81,54,108,24,12,9,9,9,12,12,12,6,12) .
\end{aligned}
$$

From Section 2, we can see that $H$ has a normal subgroup $T$ of order 108 . Since the factor group $H$ modulus $T$ is not abelian, so $H / T \cong S_{3}$, the symmetric group on three symbols. Now we obtain three irreducible characters of $H$ by lifting irreducible characters of $S_{3}$. We call these characters $\chi_{1}, \chi_{2}$, and $\chi_{3}$ and we have

$$
\begin{aligned}
& \chi_{1}=(1,1,1,1,1,1,1,1,1,1,1,1,1,1), \\
& \chi_{2}=(1,1,1,1,1,1,1,1,1,-1,-1,-1,-1,-1), \\
& \chi_{3}=(2,2,2,2,2,2,-1,-1,-1,0,0,0,0,0) .
\end{aligned}
$$

We now consider the action of $H$ on the set $\{2,3, \ldots, 13\}$. If we denote by $P$ the permutation character of this action, then we have

$$
P=(12,3,6,9,8,5,0,0,0,4,1,1,2,2)
$$

Since $\|P\|=9,\left(P, \chi_{1}\right)=2$, and $\left(P, \chi_{3}\right)=2, P-2 \chi_{1}-2 \chi_{2}$ is an irreducible character of $H$, which we denote by $\chi_{6}$. Consider the symmetric and antisymmetric parts $\chi_{\mathrm{S}}$ and $\chi_{\mathrm{A}}$ of $\chi_{6}$ (see [2, page 50]). We have

$$
\begin{aligned}
& \chi_{\mathrm{S}}=(21,3,0,6,5,2,0,0,0,3,0,0,0,3), \\
& \chi_{\mathrm{A}}=(15,6,0,3,-1,-1,0,0,0,1,1,1,0,-3) .
\end{aligned}
$$


TABLE 3.2. The character table of $H$ and its power map.

\begin{tabular}{|c|c|c|c|c|c|c|c|c|c|c|c|c|c|c|c|}
\hline & & $1 a$ & $3 a$ & $3 b$ & $3 c$ & $2 a$ & $6 a$ & $3 d$ & $9 a$ & $9 b$ & $4 a$ & $12 a$ & $12 b$ & $6 b$ & $2 b$ \\
\hline & $2 P$ & $1 a$ & $3 a$ & $3 b$ & $3 c$ & $1 a$ & $3 c$ & $3 d$ & $9 a$ & $9 b$ & $2 a$ & $6 a$ & $6 a$ & $3 b$ & $1 a$ \\
\hline & $3 P$ & $1 a$ & $1 a$ & $1 a$ & $1 a$ & $2 a$ & $2 a$ & $1 a$ & $3 a$ & $3 a$ & $4 a$ & $4 a$ & $4 a$ & $2 b$ & $2 b$ \\
\hline$\chi_{1}$ & & 1 & 1 & 1 & 1 & 1 & 1 & 1 & 1 & 1 & 1 & 1 & 1 & 1 & 1 \\
\hline$x_{2}$ & & 1 & 1 & 1 & 1 & 1 & 1 & 1 & 1 & 1 & -1 & -1 & -1 & -1 & -1 \\
\hline$x_{3}$ & & 2 & 2 & 2 & 2 & 2 & 2 & -1 & -1 & -1 & 0 & 0 & 0 & 0 & 0 \\
\hline$\chi_{4}$ & & 3 & 3 & 3 & 3 & -1 & -1 & 0 & 0 & 0 & 1 & 1 & 1 & -1 & -1 \\
\hline$\chi_{5}$ & & 3 & 3 & 3 & 3 & -1 & -1 & 0 & 0 & 0 & -1 & -1 & -1 & 1 & 1 \\
\hline$\chi_{6}$ & & 6 & -3 & 0 & 3 & 2 & -1 & 0 & 0 & 0 & 2 & -1 & -1 & 0 & 0 \\
\hline$x_{7}$ & & 6 & -3 & 0 & 3 & 2 & -1 & 0 & 0 & 0 & -2 & 1 & 1 & 0 & 0 \\
\hline$\chi_{8}$ & & 6 & -3 & 0 & 3 & -2 & 1 & 0 & 0 & 0 & 0 & $\sqrt{3}$ & $-\sqrt{3}$ & 0 & 0 \\
\hline$\chi_{9}$ & & 6 & -3 & 0 & 3 & -2 & 1 & 0 & 0 & 0 & 0 & $-\sqrt{3}$ & $\sqrt{3}$ & 0 & 0 \\
\hline$x_{10}$ & & 8 & -1 & 2 & -4 & 0 & 0 & -1 & 2 & -1 & 0 & 0 & 0 & 0 & 0 \\
\hline$x_{11}$ & & 8 & -1 & 2 & -4 & 0 & 0 & -1 & -1 & 2 & 0 & 0 & 0 & 0 & 0 \\
\hline$x_{12}$ & & 8 & -1 & 2 & -4 & 0 & 0 & 2 & -1 & -1 & 0 & 0 & 0 & 0 & 0 \\
\hline$x_{13}$ & & 12 & 3 & -3 & 0 & 0 & 0 & 0 & 0 & 0 & 0 & 0 & 0 & -1 & 2 \\
\hline$x_{14}$ & & 12 & 3 & -3 & 0 & 0 & 0 & 0 & 0 & 0 & 0 & 0 & 0 & 1 & -2 \\
\hline
\end{tabular}

In addition, $\left\|\chi_{\mathrm{S}}\right\|=4$ and $\left\|\chi_{\mathrm{A}}\right\|=2$. Since $\left(\chi_{\mathrm{S}}, \chi_{1}\right)=1,\left(\chi_{\mathrm{S}}, \chi_{3}\right)=1$, and $\left(\chi_{\mathrm{S}}, \chi_{6}\right)=1, \chi_{\mathrm{S}}-\chi_{1}-\chi_{3}-\chi_{6}$ is an irreducible character of $H$, which we denote by $\chi_{14}$. On the other hand, $\left\|\chi_{\mathrm{A}}\right\|=2$ and $\left(\chi_{\mathrm{A}}, \chi_{14}\right)=1$, so $\chi_{\mathrm{A}}-\chi_{14}$ is an irreducible character of $H$, which is denoted by $\chi_{4}$. Now we define $\chi_{7}=\chi_{6} \chi_{2}$, $\chi_{5}=\chi_{4} \chi_{2}$, and $\chi_{13}=\chi_{14} \chi_{2}$, then we obtain three irreducible characters of $H$ which are different from $\chi_{1}, \chi_{2}, \chi_{3}, \chi_{6}$, and $\chi_{14}$.

Finally we consider the character $\psi=\chi_{5} \chi_{6}$. Then $\|\psi\|=3$ and $\left(\psi, \chi_{7}\right)=1$. So, $\psi-\chi_{7}$ is a sum of two irreducible characters. Since the square of character degrees is the order of the group and the number of irreducible characters of degree $n \geq 3$ is even, $\psi-\chi_{7}=\chi_{8}+\chi_{2} \chi_{8}$, in which $\chi_{8}$ is an irreducible character of $H$. Thus, we obtain two new irreducible characters $\chi_{8}$ and $\chi_{9}=\chi_{2} \chi_{8}$. Using orthogonality relations, we have

$$
\begin{aligned}
& x_{8}=(6,-3,0,3,-2,1,0,0,0,0, \sqrt{3},-\sqrt{3}, 0,0), \\
& \chi_{9}=(6,-3,0,3,-2,1,0,0,0,0,-\sqrt{3}, \sqrt{3}, 0,0) .
\end{aligned}
$$

Using the character $\chi_{6} \chi_{14}$ and the powers of $\chi_{2}$, we can obtain other irreducible characters. We summarize our calculations in Table 3.2, the character table of the group $H$. As we mentioned above, since $G \cong Z_{2} \times H$, we can compute the character table of $G$ from Table 3.2.

ACKNOWLEDGMENT. We are greatly indebted to the referee whose valuable suggestions led us to rearrange the note. 


\section{REFERENCES}

[1] Ph. R. Bunker, Molecular Symmetry and Spectroscopy, Academic Press, New York, 1979.

[2] I. M. Isaacs, Character Theory of Finite Groups, Pure and Applied Mathematics, no. 69, Academic Press, New York, 1976.

[3] G. James and M. Liebeck, Representations and Characters of Groups, Cambridge Mathematical Textbooks, Cambridge University Press, Cambridge, 1993.

[4] J. S. Lomont, Applications of Finite Groups, Academic Press, New York, 1959.

[5] H. C. Longuet-Higgins, The symmetry groups of non-rigid molecules, Molecular Phys. 6 (1963), 445-460.

[6] Y. G. Smeyers, Introduction to group theory for non-rigid molecules, Adv. Quantum. Chem 24 (1991), no. 1, 1-77.

[7] Y. G. Smeyers (ed.), Structure and Dynamics of Non-Rigid Molecular Systems, Kluwer Academic Publishers, Dordrecht, 1995.

[8] Y. G. Smeyers, M. L. Senent, V. Botella, and D. C. Moule, An ab initio structural and spectroscopic study of acetone-an analysis of the far infrared torsional spectra of acetone-h6 and -d6, J. Chem. Phys. 98 (1993), no. 4, 2754-2767.

[9] Y. G. Smeyers and M. Villa, A study of the internal dynamics of trimethylamine by means of the non-rigid group theory, J. Math. Chem. 28 (2000), no. 4, 377-388.

[10] Y. G. Smeyers, M. Villa, and M. L. Senent, Ab initio determination of the torsionwagging and wagging-bending infrared band structure spectrum of methylamine, J. Mol. Spectrosc. 191 (1998), no. 2, 232-238.

[11] A. J. Stone, Computation of character tables for non-rigid molecules, J. Chem. Phys. 41 (1964), 1568-1579.

[12] Ad van der Avoird, van der Waals rovibration levels and the high resolution spectrum of the argon-benzene dimer, J. Chem. Phys. 98 (1993), no. 7, 53275336.

[13] A. Vivier-Bunge, V. H. Uc, and Y. G. Smeyers, Two-dimensional treatment of the methyl and aldehydic torsions in gas phase propanal, J. Chem. Phys. 109 (1998), no. 6, 2279-2286.

Masood Hamadanian: Department of Chemistry, Faculty of Science, University of Kashan, Kashan, Iran

E-mail address: hamadani@kashanu.ac.ir

Ali Reza Ashrafi: Department of Mathematics, Faculty of Science, University of Kashan, Kashan, Iran

E-mail address: ashrafi@kashanu.ac.ir 


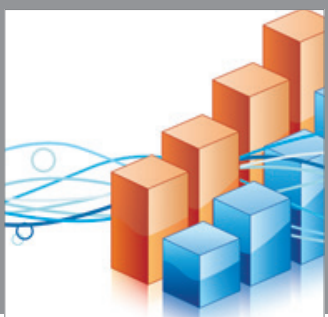

Advances in

Operations Research

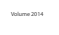

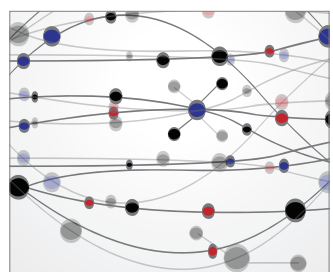

\section{The Scientific} World Journal
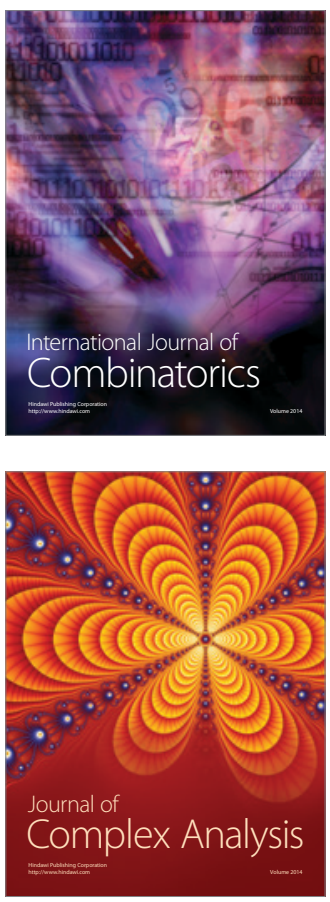

International Journal of

Mathematics and

Mathematical

Sciences
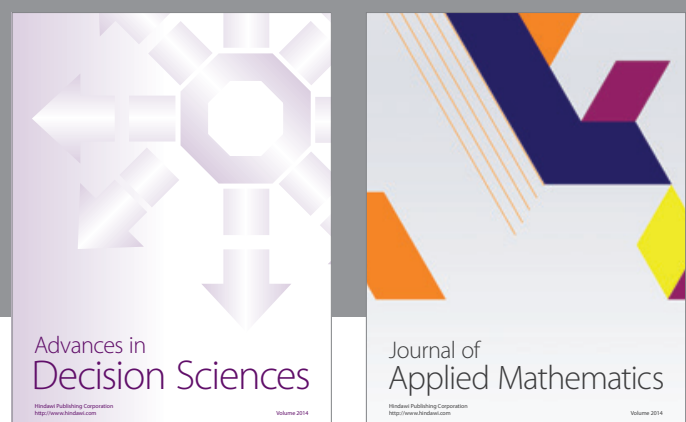

Journal of

Applied Mathematics
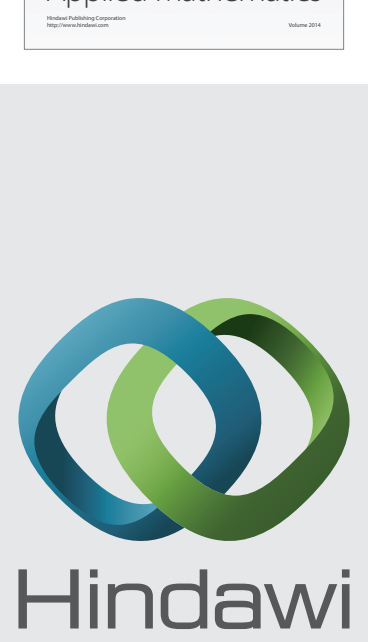

Submit your manuscripts at http://www.hindawi.com
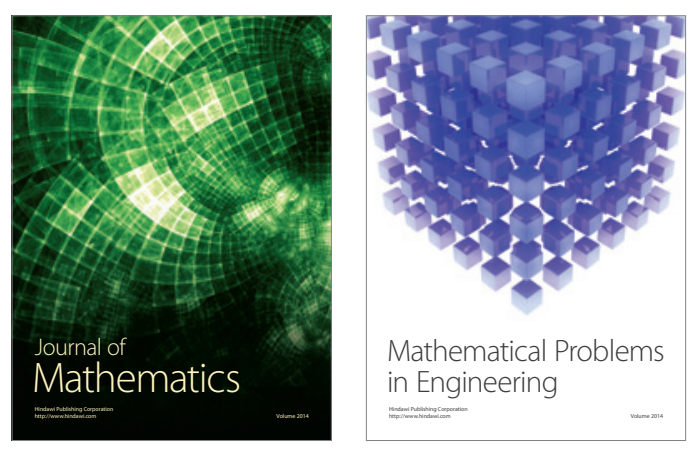

Mathematical Problems in Engineering
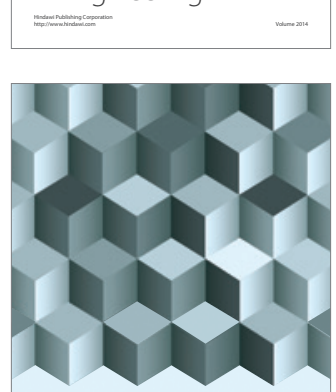

Journal of

Function Spaces
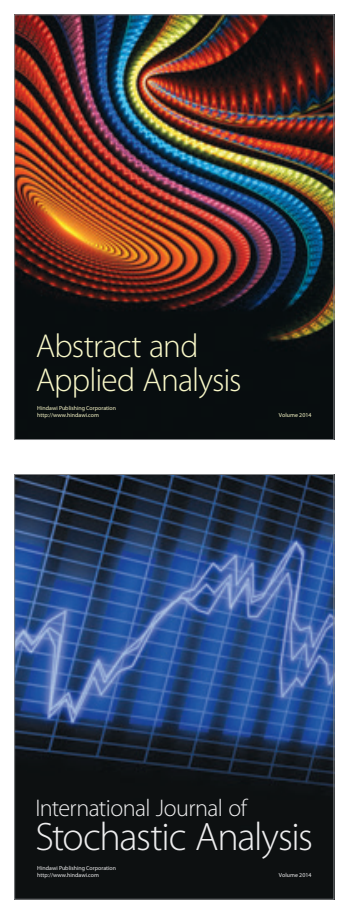

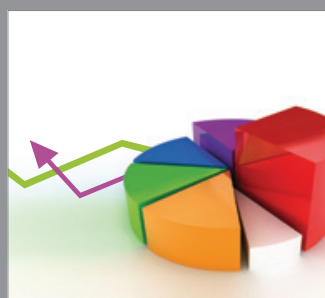

ournal of

Probability and Statistics

Promensencen
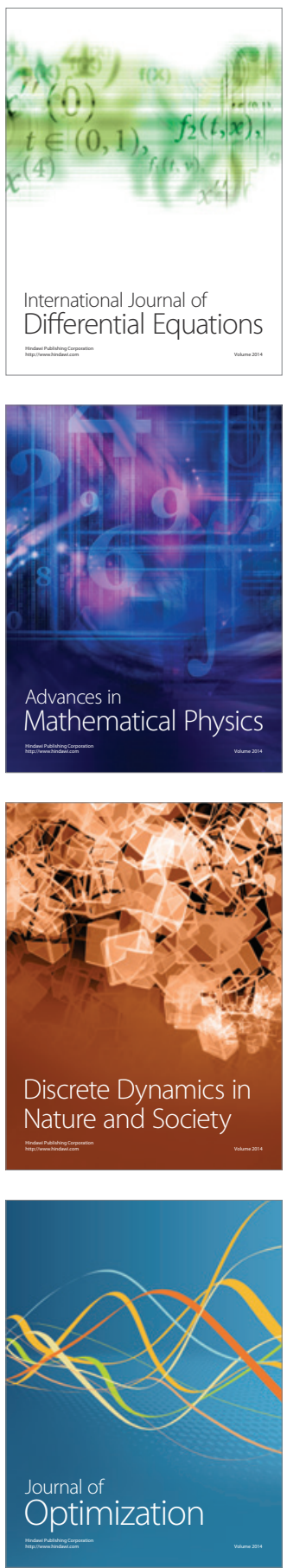